\title{
HOXB7 as a promising molecular marker for metastasis in cancers: a meta-analysis
}

This article was published in the following Dove Press journal:

OncoTargets and Therapy

6 May 2016

Number of times this article has been viewed

\author{
Fang-teng Liu \\ Yang-xi Ou \\ Guan-ping Zhang \\ Cheng Qiu \\ Hong-liang Luo \\ Pei-qian Zhu
}

Department of Gastrointestinal Surgery, the Second Affiliated Hospital of Nanchang University,

Nanchang, Jiangxi Province, People's

Republic of China
Correspondence: Hong-liang Luo;

Pei-qian Zhu

Department of Gastrointestinal Surgery, the Second Affiliated Hospital of

Nanchang University, I Minde Road,

Nanchang 330000, Jiangxi Province,

People's Republic of China

Email517295653@qq.com;

zhupeiqian@163.com

\begin{abstract}
Numerous studies on carcinoma have revealed that the expression level of $H O X B 7$ in cancerous tissues was significantly higher than that in noncancerous tissues. Elevated expression of $H O X B 7$ is associated with the susceptibility to lymph node metastasis and distant metastasis in various tumors. In this study, a meta-analysis was performed to involve majority of relevant articles and explore the association of $\mathrm{HOXB7}$ expression level with metastasis in cancer patients. Literature retrieval was conducted by searching in a number of electronic databases (up to December 1, 2015). The meta-analysis was conducted with RevMan 5.3 software and Stata SE12.0. A total of 1,532 patients with carcinoma from 14 studies were included in analysis. The results of meta-analysis demonstrated that lymph node metastasis was observed more frequently in the patients group with high expression level of $H O X B 7$ than in the patients group with low expression level of $H O X B 7$ (odds ratio $=2.17,95 \%$ CI: 1.74-2.71, $P<0.00001$, fixed-effects model). In addition, a similar result was observed in the association between HOXB7 expression and distant metastasis; the odds ratio was 1.77 (95\% CI: $1.09-2.88, P=0.02$, fixed-effects model). This meta-analysis demonstrated that the overexpression of $H O X B 7$ was significantly associated with metastasis in cancer patients, which may be served as a common molecular marker for indicating cancer metastasis.
\end{abstract}

Keywords: homeobox gene, $H O X B 7$, carcinoma, metastasis, meta-analysis

\section{Introduction}

Nowadays, cancer is a leading cause of mortality. According to a recent survey, 8.2 million people die from cancer each year and 14.1 million new cases were diagnosed with cancer worldwide. ${ }^{1}$ A majority of the cancer cases can ultimately develop metastases, which included lymph node metastases (LNM) and distant metastases (DM). The occurrence of metastasis was a critical indicator for survival, which indicated poor prognosis in most cancers. ${ }^{2,3}$ In addition, the treatment measures were also determined by whether there was metastasis or not. Until now, the precise mechanism on metastasis in cancer cells is still unclear. In recent years, molecular biomarkers, as a hotspot in cancer research, have raised a revolution in the prediction and treatment of cancer. ${ }^{4-6}$ Until now, we still know nothing about the role of $H O X B 7$ in predicting metastases of various cancers, although it may act as a common molecular marker for both LNM and DM.

$H O X$ genes belong to an important component of superfamily of homeobox genes. A large family of transcriptional factors were encoded, and the expression of many downstream target genes was regulated by $H O X$ genes. ${ }^{7,8}$ Human $H O X$ genes can be divided into two classes. In class I, there were four paralogous clusters of $H O X$ genes, and they were defined as $H O X A, H O X B, H O X C$, and $H O X D$, which were arranged in $2,12,17$, and 7 chromosomes in turn..$^{9-11}$ Many studies have reported that $H O X$ genes 
were frequently deregulated in cancers, which could widely make effects on cellular functions, including proliferation, differentiation, and apoptosis. ${ }^{12-15} \mathrm{HOX}$ genes were also involved in tumor initiation and progression. ${ }^{16,17}$

$H O X B 7$, as one of class I $H O X$ genes, was found to participate in the process of various cancers, such as gastric cancer, breast cancer (BC), and pancreatic carcinoma. ${ }^{18-20}$ Some studies revealed that $H O X B 7$ played a crucial role in tumorigenesis and was closely related to the viability, invasion, and metastasis of tumor cells. ${ }^{21,22}$ A number of studies have demonstrated that the expression level of $H O X B 7$ was upregulated in cancerous tissues and was associated with some clinical features, including LNM and DM..$^{23-25}$ Therefore, in this study, we collected relevant literatures and performed the meta-analysis. It aimed to explore the relationship between the $H O X B 7$ expression and metastasis and further determine whether $H O X B 7$ could be applied as a putative biomarker for indicating metastasis in cancer patients.

\section{Methods}

\section{Literatures' retrieval strategy}

For obtaining potentially eligible studies, integrated online literature retrieval was performed against multiple databases, including PubMed, Springer, Google Scholar, China National Knowledge Infrastructure (CNKI), Chongqing VIP Information Network, and Wanfang. The deadline of retrieval period was up to December 1, 2015. The keywords for the search were as follows: "Homeobox B7", "HOXB7”, "cancer", "carcinoma", and "neoplasm". In addition, other relevant articles were also obtained by manually viewing the reference list.

\section{Inclusion and exclusion criteria}

Inclusion criteria for the articles were as follows: 1) the role of $H O X B 7$ in the development of human cancer was investigated; 2) related clinicopathologic parameters were described; 3) the expression level of $H O X B 7$ in primary cancerous tissue was measured; and 4) patients were grouped according to the expression level of $H O X B 7$.

Exclusion criteria for the articles were as follows: 1) duplicate publications; 2) studies without valuable data; and 3 ) reviews, letters, case reports, and expert opinions.

\section{Date extraction}

The data and information from all eligible studies were independently extracted by two investigators (YX O and GP Z). The following data and information were collected from each study: author, publication year, country, race, cancer type, total number of patients, number of high $\mathrm{HOXB7}$ expression group and low $H O X B 7$ expression group, number of patients with LNM and DM in each group, and the criteria of high HOXB7 expression. If there were disagreements, a consensus was reached by a third investigator (CQ).

\section{Statistical methods}

The present meta-analysis was conducted using RevMan5.3 software and Stata SE12.0. The heterogeneity among eligible studies was determined with the chi-square-based $Q$-test and $I^{2}$ statistics; a $P$-value for $Q$-test $<0.05$ and $I^{2}$-value $>50 \%$ were considered severe heterogeneity. The random-effects model was applied in studies with a significant heterogeneity $\left(P_{\mathrm{Q}} \leq 0.05, I^{2} \geq 50 \%\right)$; otherwise, the fixed-effects model was adopted $\left(P_{\mathrm{Q}}>0.05, I^{2}<50 \%\right)$. Potential publication bias was assessed with a funnel plot, and sensitivity analysis was also performed to ensure the reliability of results. The $P$-value $<0.05$ was considered statistically significant.

\section{Result Studies' characteristics}

The process of literature retrieval is shown in detail in Figure 1. A total of 14 studies were finally identified to be eligible according to the criteria for selection. ${ }^{23-36} \mathrm{~A}$ total of 1,532 patients were included in the current meta-analysis, and the mean sample size of patients was 109.4 (range: 35-280). Among the 14 studies, 12 were from the People's Republic of China, two were from Brazil, and one was from the USA. Eight different cancer types were evaluated in this meta-analysis, with two BC, three esophagus cancer, one gastric cancer, one lung cancer, two pancreatic cancer, two colorectal cancer, two oral cancer, and one malignant ovarian germ cell tumor. All cancerous specimens were well preserved before RNA extraction. The diagnoses of LNM and DM were all based on pathology.

There were two studies that reported on the association between the expression of HOXB7 mRNA and LNM, ${ }^{23,29}$ and the rest 12 articles have paid attention on the association of HOXB7 protein expression and metastases. Two detection methods (reverse transcription polymerase chain reaction and immunohistochemical staining) were applied to determine the expression levels of $H O X B 7$ in cancerous tissues. The criteria of high $H O X B 7$ expression in all included studies are shown in Table 1. All studies were divided into two groups (high $H O X B 7$ expression group and low $H O X B 7$ expression group). For unifying the result for further analysis, positive expression of $H O X B 7$ was regarded as high expression and negative expression of $H O X B 7$ was classified into low expression group. 


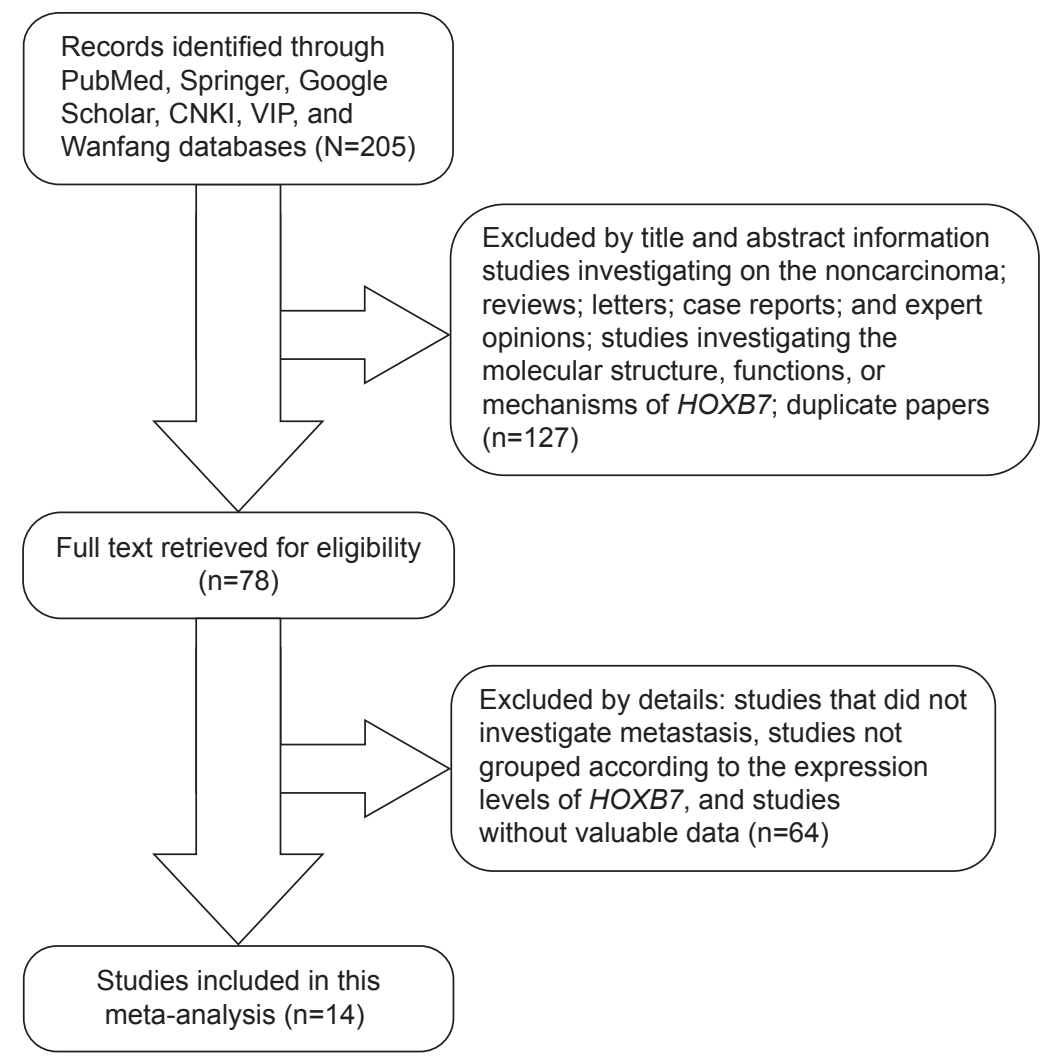

Figure I A flowchart presenting the steps of literature retrieval and selection.

Abbreviations: CKNI, China National Knowledge Infrastructure; VIP, Chongqing VIP Information Network.

\section{Association between HOXB7 expression level and LNM}

All 14 studies provided the number of patients with LNM based on different $H O X B 7$ expression levels in a total of 1,532 patients. The fixed-effects model was adopted as there was limited heterogeneity across studies $\left(I^{2}=0 \%, P_{\mathrm{Q}}=0.59\right)$. The odds ratio (OR), expressed as high $H O X B 7$ expression group versus low $\mathrm{HOXB7}$ expression group, was $2.17(95 \%$ CI: 1.74-2.71, $P<0.00001$; Figure 2). In the subgroup analysis, the result showed that there was a significant association between the expression level of HOXB7 mRNA and LNM $(\mathrm{OR}=2.22,95 \% \mathrm{CI}: 1.26-3.91, P=0.006)$. For the association between the expression level of $H O X B 7$ protein and $\mathrm{LNM}$, the $\mathrm{OR}$, expressed as high $H O X B 7$ protein expression group versus low $H O X B 7$ protein expression group, was 2.16 (95\% CI: $1.70-2.76, P<0.00001)$. From the analysis results, when the LNM incidence of cancers was compared between the two groups, we found that there was a significant difference in the LNM incidence between the high and low expression groups. This result demonstrated that cancer patients determined with high $\mathrm{HOXB} 7$ expression (including mRNA and protein) in cancerous tissues were more prone to developing LNM.

\section{Association between HOXB7 expression level and DM}

Five studies reported the number of patients with DM based on different $H O X B 7$ protein expression levels in a total of 544 patients. There was no significant heterogeneity among the studies $\left(I^{2}=15 \%, P_{\mathrm{O}}=0.32\right)$; thus, the fixed-effects model was adopted. Analysis showed a pooled OR $=1.77$ (95\% CI: 1.09-2.88, $P=0.02$; Figure 3). Compared with the low HOXB7 expression group, the DM rate was significantly increased in the high $H O X B 7$ expression group. The result showed that patients with high $H O X B 7$ protein expression level in tumor tissues may indicate an increased risk of developing DM.

In addition, sensitivity analysis and assessment of publication bias were not performed due to the relatively small heterogeneity across studies in distant metastasis and limited number of included studies.

\section{Publication bias}

For meta-analysis of the association between $\mathrm{HOXB} 7$ expression level and LNM, the funnel plot was slightly asymmetrical (Figure 4), and then, the trim and fill method was applied to test for publication bias. The results showed that there was no significant publication bias across these studies. 


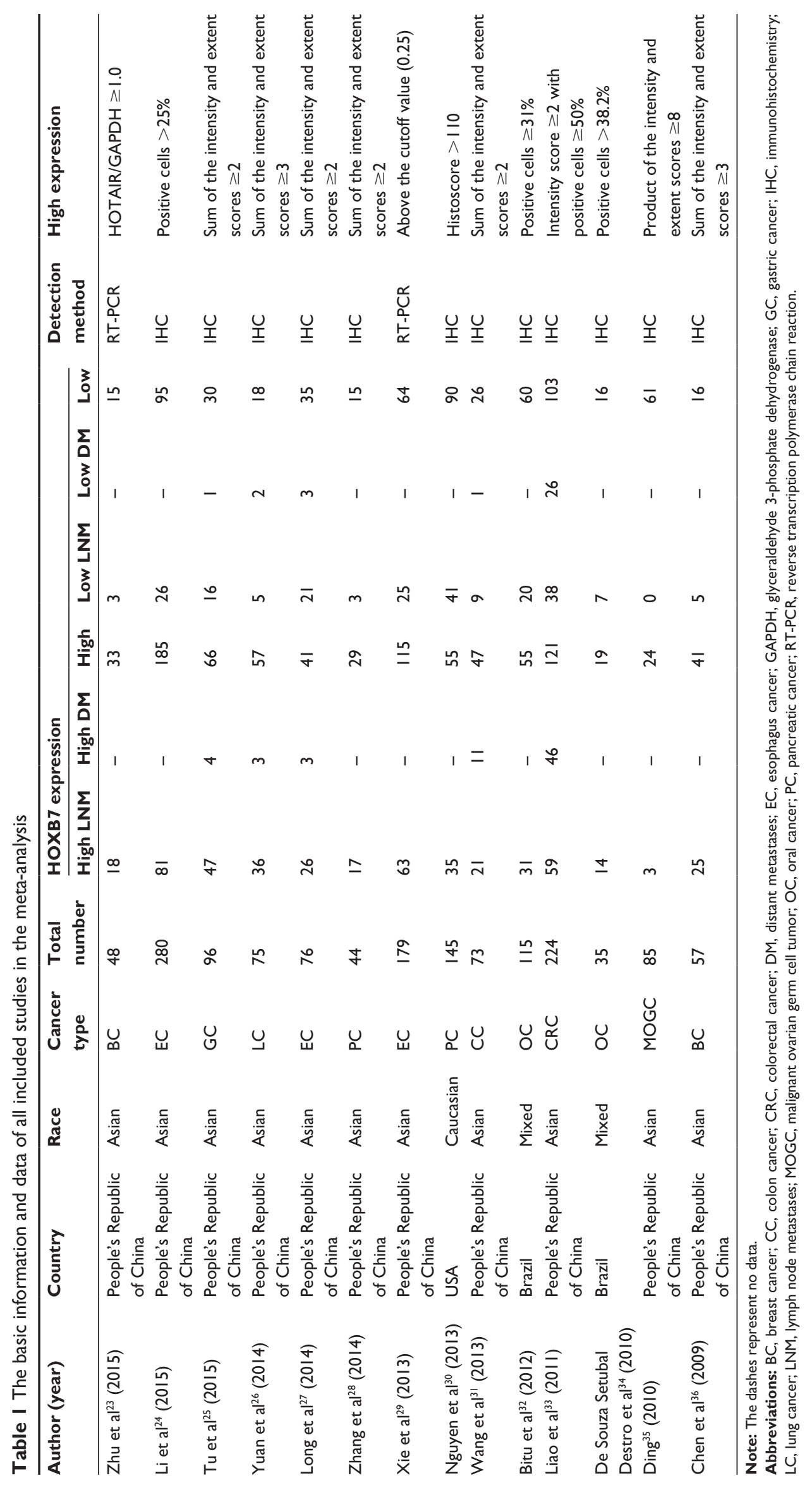




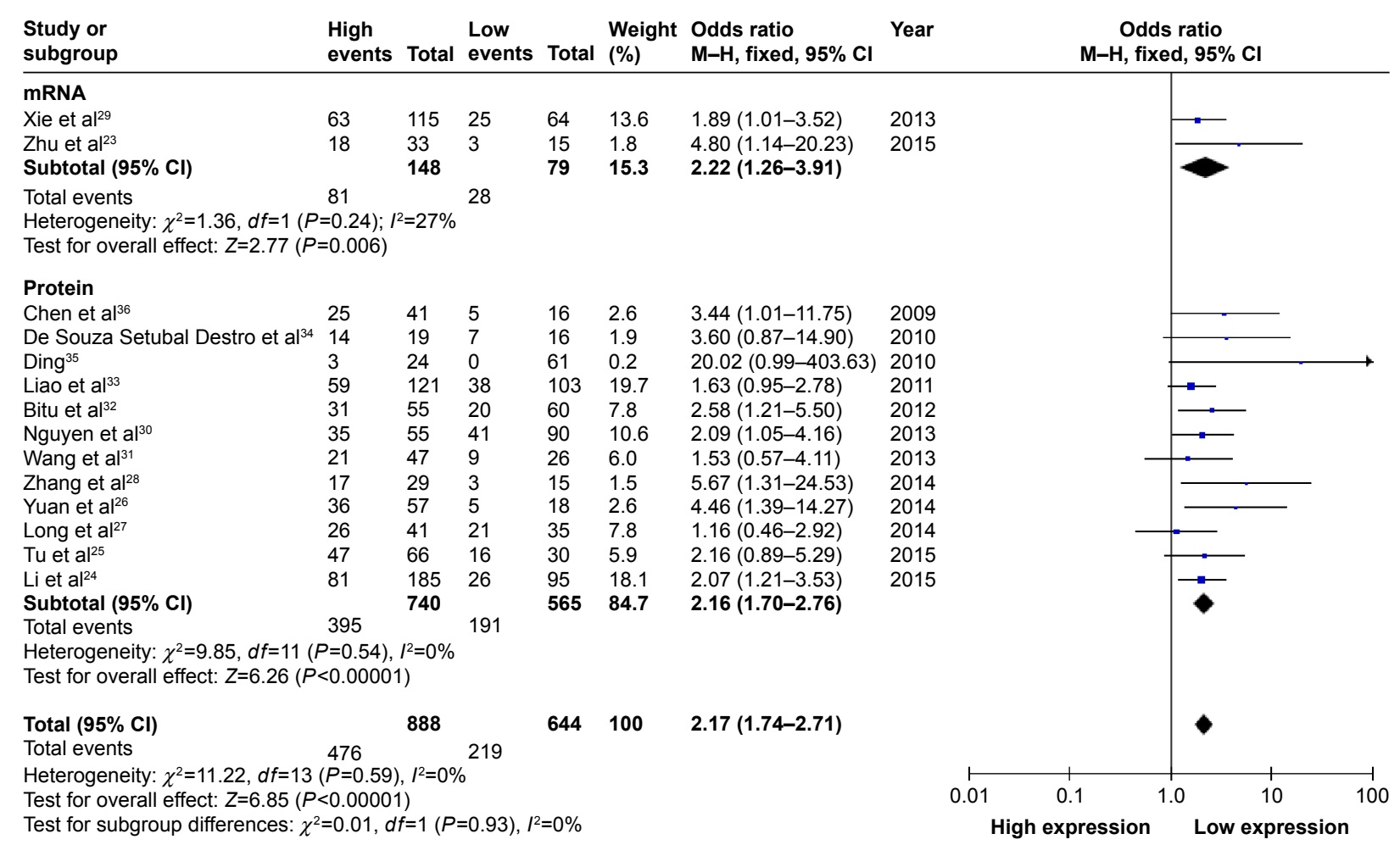

Figure 2 A forest plot for the association between HOXB7 expression levels with LNM.

Abbreviations: $d f$, degrees of freedom; LNM, lymph node metastases; $M-H$, Mantel-Haenszel test.

\section{Sensitivity analysis}

For meta-analysis of the association between $\mathrm{HOXB7}$ expression level and LNM, sensitivity analysis was performed by deleting each study in turn from the pooled analysis. It aimed to test the influence of the removed data set on the overall ORs. The result was not significantly influenced by the exclusion of each study, suggesting that the result of synthetic analysis was robust.

\section{Discussion}

As we all know, cancer is a severe threat to human health, and there are millions of people who die from cancer every year.
The LNM is the most common metastasis pathway in most cancers, and distant metastasis often occurs in the later stages of cancer. LNM and DM are positively significant for diagnosis in tumor-node-metastasis staging and treatment for cancer patients, as well as are important indicators for predicting prognosis. Thereby, further discovery of new molecular markers to predict metastasis for cancer is still essential and full of clinical significance.

A growing number of researches have showed that the expression of $H O X B 7$ was higher in cancerous tissues compared with paired noncancerous tissues. There were associations between $H O X B 7$ expression and certain clinical

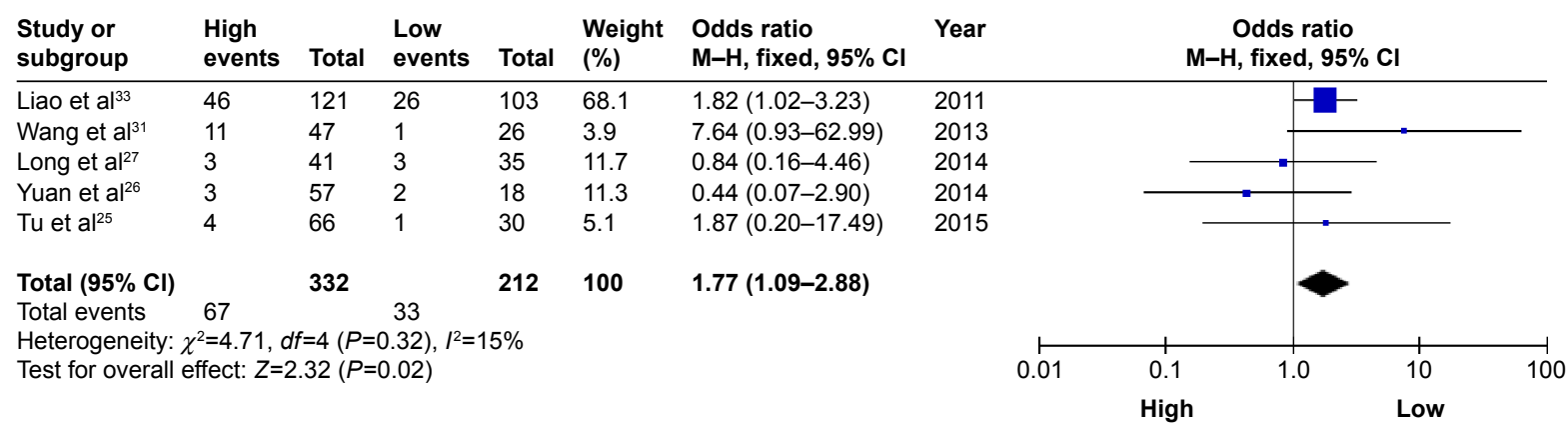

Figure $3 \mathrm{~A}$ forest plot for the association between $\mathrm{HOXB7}$ expression levels with DM. 


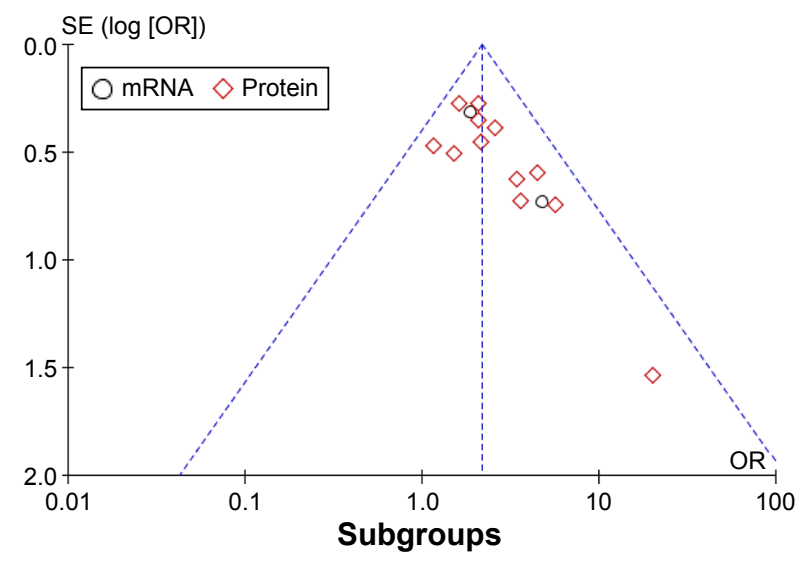

Figure $4 \mathrm{~A}$ funnel plot analysis of potential publication bias. Abbreviations: SE, standard error; OR, odds ratio.

characteristics of cancer patients. The patients with high expression levels of $H O X B 7$ had an increased risk of metastasis as well as a poor overall survival. ${ }^{26-28}$ Furthermore, the overexpression of $H O X B 7$ was closely related to the aggressive behavior of tumor cells. However, the exact mechanism of how $H O X B 7$ makes effects on promoting tumor cell invasion and metastasis has not been clear and is still in research stage. Wu et $\mathrm{al}^{37}$ found that tumor invasion in $\mathrm{BC}$ could be promoted by $H O X B 7$ through Ras/Rho pathway activation after upregulating basic fibroblast growth factor (bFGF), which was a known transcriptional target of $H O X B 7$. In the malignant melanoma, it has demonstrated that miR-196a was a central regulator of $H O X B 7$ expression, which played an important role in melanoma progression. ${ }^{38}$ Recently, a study by Liu et $\mathrm{al}^{39}$ showed that the migration and invasion of $\mathrm{BC}$ cells may be increased by overexpression of $\mathrm{HOXB7}$, and this result could be reversed by knockdown of transforming growth factor (TGF) $\beta 2$ or pharmacologic inhibition of TGF $\beta$ signaling. It suggested that $H O X B 7$ may make effects on promoting tumor malignant progression through the activation of TGF $\beta$ signaling pathway. ${ }^{42-44} H O X B 7$ was also found to promote the migration and metastasis in lung adenocarcinoma through activation of the TGF $\beta /$ SMAD3 signaling. ${ }^{40}$ Chile et $\mathrm{al}^{41}$ reported that $H O X B 7$ was also involved in cell proliferation and viability. Cell cycle arrest and apoptosis could be induced by the knockdown of $H O X B 7$ in pancreatic ductal adenocarcinomas, while decreased protein level could significantly lead to the increased apoptosis rate. $H O X B 7$ may be a promising target for future cancer therapies.

\section{Conclusion}

This meta-analysis has explored the relation between $\mathrm{HOXB7}$ expression levels with LNM and DM for carcinoma. From the results of this current meta-analysis, we found that the occurrence probability of LNM and DM was higher in cancer patients with high $H O X B 7$ expression, comparing with that of those with low $H O X B 7$ expression. Nevertheless, we also found that there were some limitations in this meta-analysis. Firstly, the included number of cancer patients has been still relatively small, hence larger and better design studies would be necessary to confirm the obtained results. Besides, most patients included in this meta-analysis have been Asian, the patients of other races accounted for a small percentage. Additionally, potential publication bias may exist, although no significant publication bias was observed based on trim and fill method and the sensitivity analysis have also showed the results were robust. Furthermore, the criterion of high HOXB7 expression was varied in these studies. Therefore, more larger size, multicenter, and higher quality studies are needed for further research, based on a unified criterion for classifying $H O X B 7$ expression groups.

\section{Author contributions}

H-L Luo and P-Q Zhu were involved in the design of this meta-analysis and revision of this manuscript; they have also given final approval for submission. Y-X Ou, G-P Zhang, and C Qiu were involved in publication collection. F-T Liu was involved in data analysis of the study and manuscript writing. He has also assisted in the design of this work. All authors contributed toward data analysis, drafting and critically revising the paper and agree to be accountable for all aspects of the work.

\section{Disclosure}

The authors report no conflicts of interest in this work.

\section{References}

1. Ferlay J, Soerjomataram I, Dikshit R, et al. Cancer incidence and mortality worldwide: sources, methods and major patterns in GLOBOCAN 2012. Int J Cancer. 2014;136(5):E359-E386.

2. Cho JH, Lee YS, Sun DI, et al. Prognostic impact of lymph node micrometastasis in oral and oropharyngeal squamous cell carcinomas. Head Neck. Epub 2015 Dec 17.

3. Li P, Wu F, Zhao H, et al. Analysis of the factors affecting lymph node metastasis and the prognosis of rectal neuroendocrine tumors. Int J Clin Exp Pathol. 2015;8(10):13331-13338.

4. Mirzaei H, Gholamin S, Shahidsales S, et al. MicroRNAs as potential diagnostic and prognostic biomarkers in melanoma. Eur J Cancer. 2015; 53:25-32.

5. Gao J, Cao R, Mu H. Long non-coding RNA UCA1 may be a novel diagnostic and predictive biomarker in plasma for early gastric cancer. Int J Clin Exp Pathol. 2015;8(10):12936-12942.

6. Treilleux I, Arnedos M, Cropet C, et al. Translational studies within the TAMRAD randomized GINECO trial: evidence for mTORC1 activation marker as a predictive factor for everolimus efficacy in advanced breast cancer. Ann Oncol. 2015;26(1):120-125.

7. Cantile M, Schiavo G, Terracciano L, Cillo C. Homeobox genes in normal and abnormal vasculogenesis. Nutr Metab Cardiovasc Dis. 2008; 18(10):651-658 
8. Samuel S, Naora H. Homeobox gene expression in cancer: insights from developmental regulation and deregulation. Eur J Cancer. 2005; 41(16):2428-2437.

9. Chen H, Sukumar S. Role of homeobox genes in normal mammary gland development and breast tumorigenesis. J Mammary Gland Biol Neoplasia. 2003;8(2):159-175.

10. Chen H, Sukumar S. HOX genes: emerging stars in cancer. Cancer Biol Ther. 2003;2(5):524-525.

11. Cillo C, Faiella A, Cantile M, Boncinelli E. Homeobox genes and cancer. Exp Cell Res. 1999;248(1):1-9.

12. Wang H, Liu G, Shen D, et al. HOXA1 enhances the cell proliferation, invasion and metastasis of prostate cancer cells. Oncol Rep. 2015;34(3): 1203-1210.

13. Seifert A, Werheid DF, Knapp SM, Tobiasch E. Role of Hox genes in stem cell differentiation. World J Stem Cells. 2015;7(3):583-595.

14. Domsch K, Papagiannouli F, Lohmann I. The HOX-apoptosis regulatory interplay in development and disease. Curr Top Dev Biol. 2015;114: 121-158.

15. Platais C, Hakami F, Darda L, Lambert DW, Morgan R, Hunter KD The role of HOX genes in head and neck squamous cell carcinoma. J Oral Pathol Med. Epub 2015 Dec 14.

16. Hamid AR, Hoogland AM, Smit F, et al. The role of HOXC6 in prostate cancer development. Prostate. 2015;75(16):1868-1876.

17. Shah N, Sukumar S. The Hox genes and their roles in oncogenesis. Nat Rev Cancer. 2010;10(5):361-371.

18. Cai JQ, Xu XW, Mou YP, Chen K, Pan Y, Wu D. Upregulation of $\mathrm{HOXB} 7$ promotes the tumorigenesis and progression of gastric cancer and correlates with clinical characteristics. Tumour Biol. Epub 2015 Aug 26.

19. Chen H, Lee JS, Liang X, et al. Hoxb7 inhibits transgenic HER-2/ neu-induced mouse mammary tumor onset but promotes progression and lung metastasis. Cancer Res. 2008;68(10):3637-3644.

20. Chile T, Fortes MA, Corrêa-Giannella ML, et al. HOXB7 mRNA is overexpressed in pancreatic ductal adenocarcinomas and its knockdown induces cell cycle arrest and apoptosis. BMC Cancer. 2013;13:451.

21. Ma R, Zhang D, Hu PC, Li Q, Lin CY. HOXB7-S3 inhibits the proliferation and invasion of MCF-7 human breast cancer cells. Mol Med Rep. 2015;12(4):4901-4908.

22. Kokonoe M, Jun-Ichi H, Minoru T, et al. Aberrant expression of HOX genes in human invasive breast carcinoma. Oncol Rep. 2005;13(4):673-679.

23. Zhu FX, Ma R, Lin CY, et al. Expression of homeobox B7(HOBX7) and its clinical significance in breast cancers. Med J Wuhan Univ. 2015; 36(1):58-61. (Chinese).

24. Li H, Shen LY, Yan WP, et al. Deregulated HOXB7 expression predicts poor prognosis of patients with esophageal squamous cell carcinoma and regulates cancer cell proliferation in vitro and in vivo. PLoS One. 2015;10(6): e0130551.

25. Tu W, Zhu X, Han Y, Wen Y, Qiu G, Zhou C. Overexpression of HOXB7 is associated with a poor prognosis in patients with gastric cancer. Oncol Lett. 2015;10(5):2967-2973.

26. Yuan W, Zhang X, Xu Y, Li S, Hu Y, Wu S. Role of HOXB7 in regulation of progression and metastasis of human lung adenocarcinoma. Mol Carcinog. 2014;53(1):49-57.

27. Long QY, Zhou J, Zhang XL, Cao JH. HOXB7 predicts poor clinical outcome in patients with advanced esophageal squamous cell cancer. Asian Pac J Cancer Prev. 2014;15(4):1563-1566.

OncoTargets and Therapy

\section{Publish your work in this journal}

OncoTargets and Therapy is an international, peer-reviewed, open access journal focusing on the pathological basis of all cancers, potential targets for therapy and treatment protocols employed to improve the management of cancer patients. The journal also focuses on the impact of management programs and new therapeutic agents and protocols on
28. Zhang R, Zheng S, Du Y, Wang Y, Zang W, Zhao G. Levels of HOXB7 and miR-337 in pancreatic ductal adenocarcinoma patients. Diagn Pathol. 2014;9(2):1-7.

29. Xie X, Zhang SS, Wen J, et al. Prognostic value of HOXB7 mRNA expression in human oesophageal squamous cell cancer. Biomarkers. 2013;18(4):297-303.

30. Nguyen Kovochich A, Arensman M, Lay AR, et al. HOXB7 promotes invasion and predicts survival in pancreatic adenocarcinoma. Cancer. 2013;119(3):529-539.

31. Wang DQ, Yan DW, Tang HM, et al. Expression of homeobox B7 gene in colon carcinoma and its clinicopathological significance. Chin J Exp Surg. 2013;30(3):438-440 (in Chinese).

32. Bitu CC, Carrera M, Lopes MA, Kowalski LP, Soares FA, Coletta RD. $\mathrm{HOXB} 7$ expression is a prognostic factor for oral squamous cell carcinoma. Histopathology. 2012;60(4):662-665.

33. Liao WT, Jiang D, Yuan J, et al. HOXB7 as a prognostic factor and mediator of colorectal cancer progression. Clin Cancer Res. 2011;17(11): 3569-3578.

34. De Souza Setubal Destro MF, Bitu CC, Zecchin KG, et al. Overexpression of HOXB7 homeobox gene in oral cancer induces cellular proliferation and is associated with poor prognosis. Int J Oncol. 2010; 36(1):141-149.

35. Ding H. The study of the expression of HOXB7 gene and its clinical significance in malignant ovarian germ-cell tumor, Master's Thesis, Sun Yat-Sen University, Guangzhou, 2010.

36. Chen ZH, LV GM, Ji TH. Expression of homeobox B7 in breast cancers and its clinical significance. China Trop Med. 2009;9(9):1697-1806 (in Chinese).

37. Wu X, Chen H, Parker B, et al. HOXB7, a homeodomain protein, is overexpressed in breast cancer and confers epithelial-mesenchymal transition. Cancer Res. 2006;66(19):9527-9534.

38. Braig S, Mueller DW, Rothhammer T, Bosserhoff AK. MicroRNA miR-196a is a central regulator of HOX-B7 and BMP4 expression in malignant melanoma. Cell Mol Life Sci. 2010;67(20):3535-3548.

39. Liu S, Jin K, Hui Y, et al. HOXB7 promotes malignant progression by activating the TGF $\beta$ signaling pathway. Cancer Res. 2015;75(4): 709-719.

40. Zhuang L, Li WH, Li K, Mao Y, Gao CL, Zhang C. Hoxb7 promotes growth and metastasis of lung adenocarcinoma cells through regulation of the tgf- $\beta /$ smad3 signaling. J Biol Regul Homeost Agents. 2015;29(3):601-608.

41. Chile T, Brentani HP, Maria DA, et al. HOXB7 mRNA is overexpressed in pancreatic ductal adenocarcinomas and its knockdown induces cell cycle arrest and apoptosis. BMC Cancer. 2013;13(1):1-12.

42. Bohlius J, Schmidlin KC, Schwarzer G, et al. Recombinant human erythropoiesis-stimulating agents and mortality in patients with cancer: a meta-analysis of randomised trials. Lancet. 2009;373(9674): $1532-1542$.

43. Yan S, Jiao X, Li K, Li W, Zou H. The impact of IGF-1R expression on the outcomes of patients with breast cancer: a meta-analysis. Onco Targets Ther. 2015;8:279-287.

44. Chen YY, Wang LW, Wang SY, et al. Meta-analysis of postoperative adjuvant chemotherapy without radiotherapy in early stage non-small cell lung cancer. Onco Targets Ther. 2015;8:2033-2043.

patient perspectives such as quality of life, adherence and satisfaction The manuscript management system is completely online and includes a very quick and fair peer-review system, which is all easy to use. Visit http://www.dovepress.com/testimonials.php to read real quotes from published authors.

\section{Dovepress}

